\title{
Analysis of a Real-Time Separation Assurance System with Integrated Time-in-Trail Spacing
}

\author{
Arwa S. Aweiss, ${ }^{*}$ Amir H. Farrahi, ${ }^{\dagger}$ Todd A. Lauderdale, ${ }^{\ddagger}$ Adam S. Thipphavong ${ }^{\S}$, and Chu H. Lee ${ }^{* *}$ \\ NASA Ames Research Center, Moffett Field, CA 94035
}

\begin{abstract}
This paper describes the implementation and analysis of an integrated ground-based separation assurance and time-based metering prototype system into the Center-TRACON Automation System. The integration of this new capability accommodates constraints in four-dimensions: position ( $x-y)$, altitude, and meter-fix crossing time. Experiments were conducted to evaluate the performance of the integrated system and its ability to handle traffic levels up to twice that of today. Results suggest that the integrated system reduces the number and magnitude of time-in-trail spacing violations. This benefit was achieved without adversely affecting the resolution success rate of the system. Also, the data suggest that the integrated system is relatively insensitive to an increase in traffic of twice the current levels.
\end{abstract}

\section{Nomenclature}

\author{
$\mathrm{AAC}=$ Advanced Airspace Concept \\ CTAS $=$ Center-TRACON Automation System \\ NextGen $=$ Next-Generation Air Transportation System \\ TRACON $=$ Terminal Radar Approach Control
}

\section{Introduction}

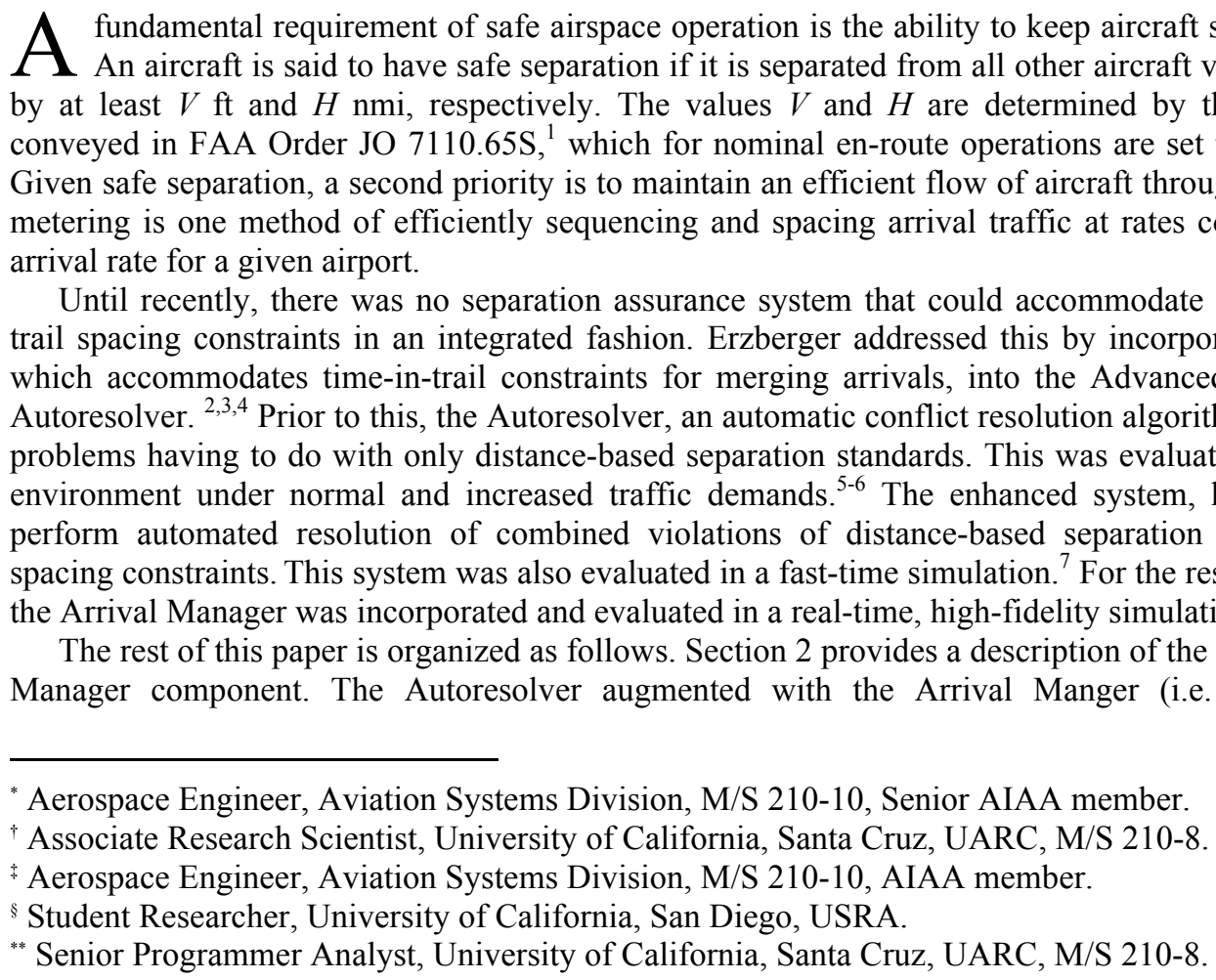


Assurance System) will be referred to as the integrated system in the rest of this paper. The experimental setup and procedures for the evaluation of the prototype system under $1 \mathrm{X}$ and $2 \mathrm{X}$ traffic levels in Fort Worth Air Route Traffic Control Center (Fort Worth Center) are presented in Section 3. A detailed analysis of the performance of the integrated system using six traffic scenarios representing nominal and highly increased demand is provided in Section 4. In the last section, a summary highlighting the key findings of this work is presented.

\section{Integrated Separation Assurance System}

Figure 1 presents a schematic of the integrated system of which the Autoresolver is central. Under the Advanced Airspace Concept, the Autoresolver is responsible for resolving traffic conflicts that are predicted to occur approximately 2 to 20 minutes in the future. There are four main modules in the integrated system; the Trajectory Synthesizer, the Conflict Probe, the First Come First Serve (FCFS) Scheduler, and the Autoresolver. The Trajectory Synthesizer sends trajectories to both the FCFS Scheduler and the Conflict Probe. The scheduler determines each aircraft's scheduled time of arrival (STA) and sends it to the Autoresolver to be used by Arrival Manager. Input for the Autoresolver also comes from the probe. The Conflict Probe algorithm generates and updates predicted conflict pairs every 12 seconds and sends these pairs along with characteristics of the conflict such as aircraft type, location, speed, and flight plan to the Autoresolver. Based on the type of conflict, these data are sent to either the Arrival Manager or the Resolution Generator. For merging-arrival conflicts, the conflict data go to the Arrival Manager, which performs arrival scheduling, sequencing, and conflict resolution in an integrated fashion. For all other conflict types, the conflict data go to the Resolution Generator algorithm.

The Autoresolver logic follows four steps. First, procedural logic determines a prioritized set of trial-resolution maneuvers that are likely to result in a successful resolution. Second, the Autoresolver uses analytical formulas and heuristics to calculate the parameters of a simplified resolution trajectory for each of the maneuvers in the set. Next, the simplified trajectory parameters are sent to the Trajectory Synthesizer that computes a trial resolution trajectory. In the fourth step, the four-dimensional (4-D) trial resolution trajectory is sent to the Conflict Probe, which determines if the trajectory solves the conflict without introducing new unintentional conflicts. If it is determined to be conflict free, it is considered a candidate resolution trajectory. If it is an arrival problem, the first conflict-free resolution trajectory is chosen as the commanded trajectory. If it is a non-arrival, the resolution process starts again using the next trial maneuver in the set. The Autoresolver stores all the viable candidate trajectories, and when all the possible maneuver resolutions have been checked, the candidate trajectory that imposes the least amount of airborne delay is chosen as the commanded trajectory. ${ }^{8}$

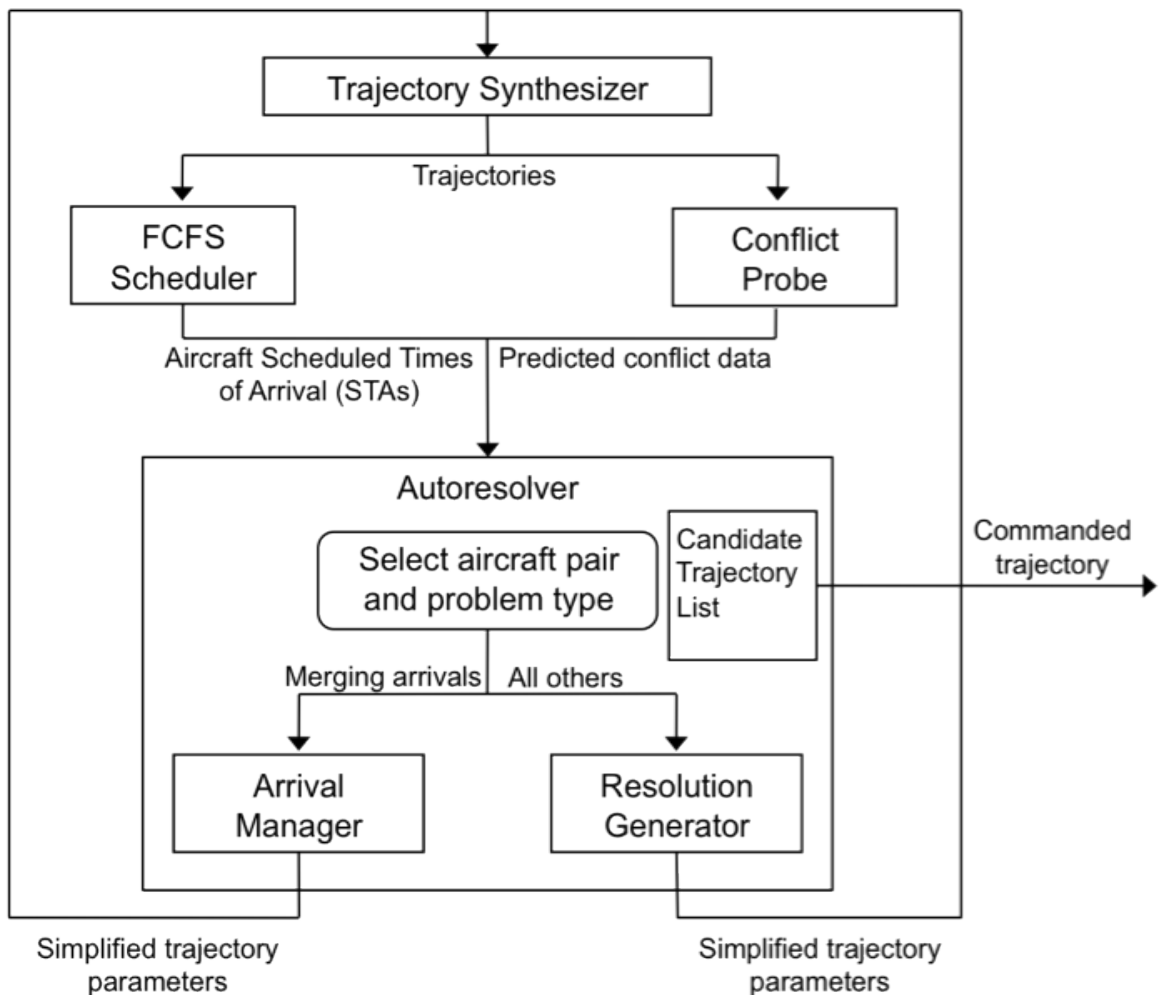

Figure 1. Integrated Separation Assurance System schematic. 
As previously stated, in an integrated separation assurance solution, both violations of distance-based separation standards as well as violations of time-in-trail spacing constraints are detected and resolved. These types are illustrated in Figs. 2 and 3. For example in Fig. 2, a predicted distance-based conflict is detected between Aircraft A and $\mathrm{B}$, meaning that the aircraft are predicted to be in violation of the distance-based separation standards. If this turns out to be a valid detection (i.e. the conflict is observed in the future), it is considered a loss of separation. For this study, the time-based requirement was modeled as a constant time-in-trail spacing constraint, which sets a lower bound on the interval between every two consecutive aircraft that cross the same arrival fix. In Fig. 3 for example, Aircraft $\mathrm{C}$ and $\mathrm{D}$ both heading to the same arrival fix, are predicted to be in violation of the time-in-trail spacing constraint. This is considered a sequencing conflict.

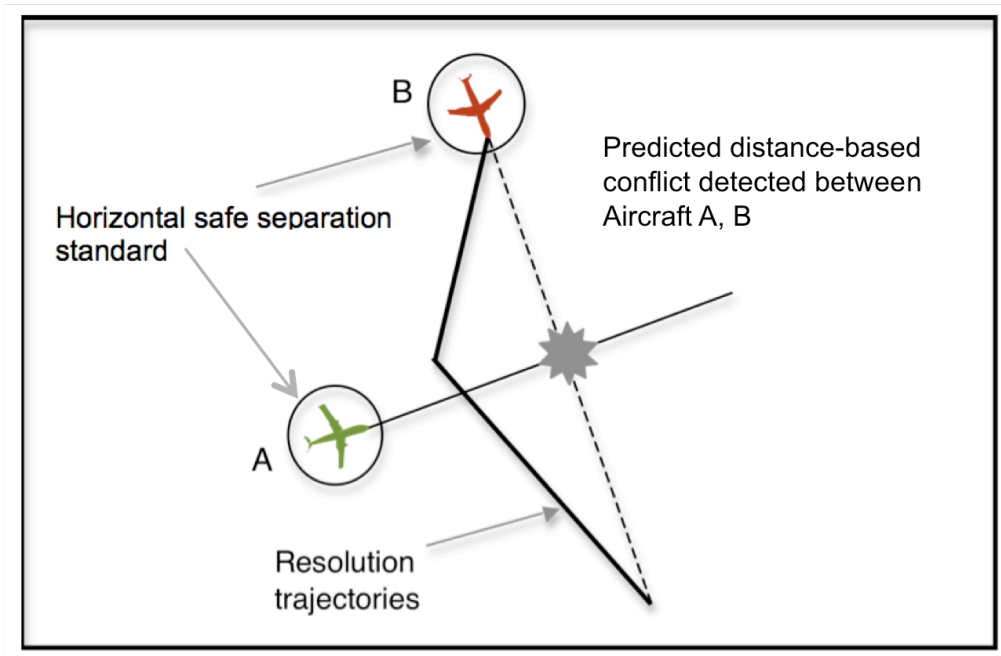

Figure 2. Predicted distance-based conflict.

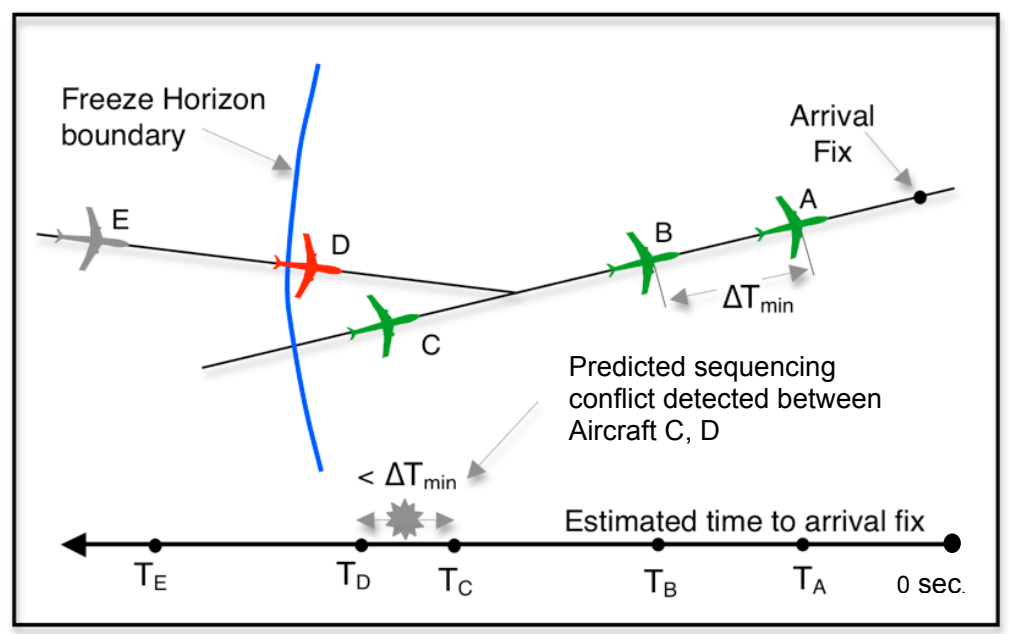

Figure 3. Predicted sequencing conflict. 


\section{Procedure}

To demonstrate and evaluate the new research capability, a "proof-of-concept" experiment was designed. A total of three 3-hour sets of traffic data were recorded from live Fort Worth Center traffic for September 10, 17, and 24, 2009. These constitute representative traffic scenarios for the baseline condition (1X). The selected scenarios included arriving, departing, and over-flight traffic. Fort Worth Center covers a very large region (roughly 121,000 $\mathrm{nmi}^{2}$ ), and it contains a single, symmetric, four-corner-post primary TRACON airspace serving the major Dallas/Fort Worth International hub airport as well as several smaller airports.

Using the baseline recorded traffic, a demand set with roughly twice the baseline traffic demand (2X) was generated in order to stress the conflict resolution algorithm and to investigate its limitations with regard to the traffic volume. The $2 \mathrm{X}$ traffic volume was generated using the methodology described by McNally and Gong, which relied upon recorded traffic to obtain flight plans and to generate initial conditions in the system. ${ }^{8}$

The simulation schematic is shown in Fig. 4. The Integrated Separation Assurance System (Fig. 1) is the test article. As previously mentioned, this consists of the Trajectory Synthesizer, the FCFS Scheduler, the Conflict Probe, and the Autoresolver. The system generates commanded trajectories (i.e. conflict-resolution trajectories) to be executed by the airspace simulator test bed. The test bed is the Center-TRACON Automation System (CTAS), which provides the real-time airspace simulation. ${ }^{9}$ Note that CTAS does not execute commanded trajectories with perfect precision; there is inherent error not associated with modeled error sources such as wind or delays.

TEST BED

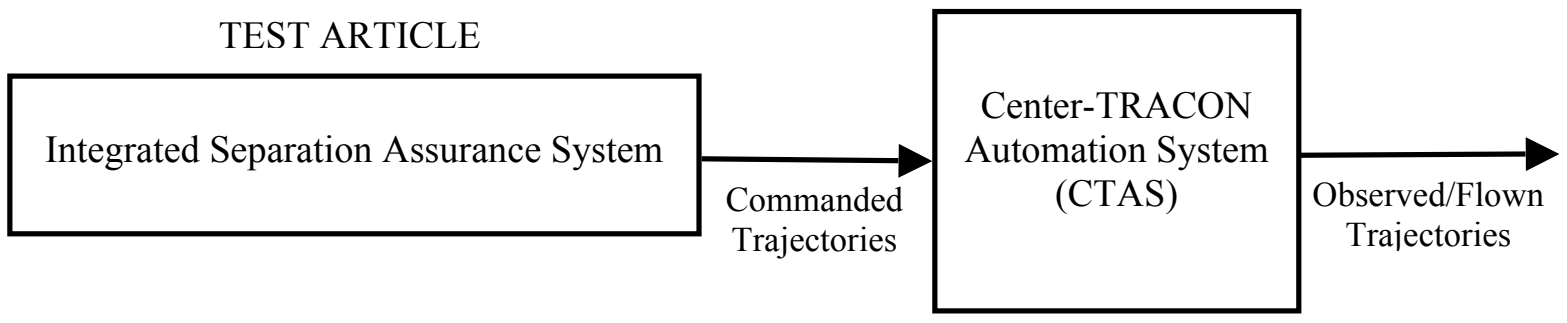

Figure 4. Simulation schematic.

The integrated system was initialized with the parameters listed in Table 1. A horizontal conflict detection criterion of seven nautical miles was enforced. As previously noted, the current-day separation standard is five nautical miles. The additional two miles were added as a buffer to account for flight trajectory uncertainty. The vertical conflict detection criterion was 1,000 feet for a pair of level aircraft and 1,500 feet for a pair of aircraft when at least one of them was in climb or descent. A minimum time-in-trail spacing constraint of one minute was used. The freeze horizon specifies the time horizon, as measured from the arrival fix, upon which arrival aircraft become eligible for sequencing and scheduling by the Arrival Manager. A freeze horizon value of 20 minutes was used, consistent with that used by Farley, et al. ${ }^{9}$

A total of twelve simulation test cases were executed as shown in Table 2. These cases expose two levels of automation for each of two levels of traffic demand (1X and 2X) for each of the three traffic samples. The levels of automation are: (a) Automated conflict detection and resolution without the integrated time-in-trail spacing capability (i.e., without Arrival Manager); and (b) Automated conflict detection and resolution with the integrated time-in-trail spacing capability (with Arrival Manager).

Table 1. AAC parameters

\begin{tabular}{|l|l|}
\hline Horizontal separation criteria (detection) & $7 \mathrm{nmi}$ \\
\hline Vertical separation criteria (level aircraft pairs) & $1000 \mathrm{ft}$ \\
\hline Vertical separation criteria (non-level) & $1500 \mathrm{ft}$ \\
\hline Freeze Horizon & $20 \mathrm{~min}$ \\
\hline Minimum time-in-trail spacing constraint & $1 \mathrm{~min}$ \\
\hline
\end{tabular}

Table 2. Test matrix

\begin{tabular}{|l|l|l|}
\hline & $1 \mathrm{X}$ & $2 \mathrm{X}$ \\
\hline Without Arrival Manger & 3 & 3 \\
\hline With Arrival Manager & 3 & 3 \\
\hline
\end{tabular}




\section{Results}

The experimental results are presented in two parts. First, the distance-based results of the integrated system are presented. These results characterize the system's performance in terms of its ability to successfully resolve distance-based conflicts. In the second part, results related to the effect of Arrival Manager on overall system performance are presented.

The following are observed results, which are based on the outcome of the real-time simulation (i.e., CTAS execution) and are the best indicator of system performance. Where applicable, the results are presented in two categories; those related to all aircraft and those related to only merging-arrival aircraft pairs. A merging-arrival pair is defined as a pair of aircraft bound for - and within the freeze horizon $(20 \mathrm{~min})$ to - the same arrival fix for the same airport.

\section{A. Distance-Based Performance of the Integrated System}

The number of aircraft and detected distance-based separation conflicts for the nine-hour simulation, categorized into merging arrivals and all other flights are shown in Table 3. Despite the fact that merging arrivals comprise less than a quarter of the aircraft present in the simulation scenarios, they account for more than half of the separation conflicts.

Table 3. Scenario summary

\begin{tabular}{|l|l|l|}
\hline & $1 \mathrm{X}$ & $2 \mathrm{X}$ \\
\hline Flights (9 hours) & 3,404 & 6,272 \\
\hline - Merging arrivals & 769 & 1,472 \\
\hline - All others & 2,635 & 4,800 \\
\hline Total conflicts & 932 & 2,931 \\
\hline - Merging arrival conflicts & 527 & 1,568 \\
\hline - All other conflicts & 405 & 1,363 \\
\hline
\end{tabular}

Figure 5 shows the resolution success rate for all aircraft as well as for only merging arrivals at both $1 \mathrm{X}$ and $2 \mathrm{X}$ traffic. The resolution success rate is calculated by the number of resolved conflicts, as determined from CTAS track data output, over the corresponding number of detected conflicts listed in Table 3 . The integrated system had over a $96 \%$ and $94 \%$ resolution success rate in $1 \mathrm{X}$ and $2 \mathrm{X}$ traffic, respectively. Although the number of conflicts more than tripled from $1 \mathrm{X}$ to $2 \mathrm{X}$ traffic, predicted conflict resolution performance decreased by a modest two percent. This suggests that the algorithm is relatively insensitive to increases in traffic density up to $2 \mathrm{X}$.

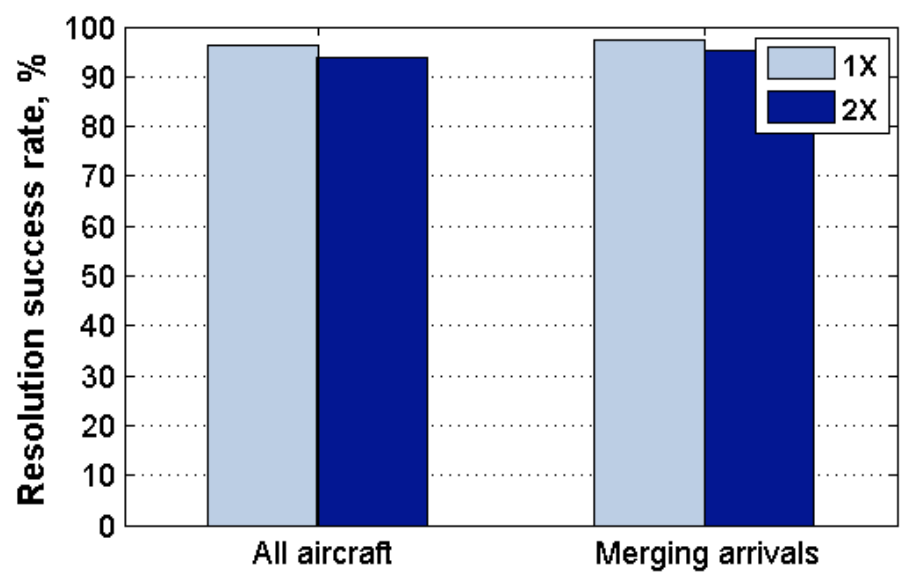

Figure 5. Distance-based resolution success rates. 
There are six conflict resolution types that are available; Direct-to, Altitude, Analytical Turn, Route Off-Set, Path Stretch, and Speed. For arrivals however, Direct-to is unavailable and the preferred resolution types are speed and path stretch changes. Speed profile changes are tried first, and if those fail path stretches are next. The first resolution trajectory that meets the required time-in-trail spacing is chosen. More details on resolution type selection can be found in Erzberger. ${ }^{4}$ For this dataset, in both $1 \mathrm{X}$ and $2 \mathrm{X}$ traffic, the integrated system was able to identify more than one acceptable resolution for a high percentage (over $90 \%$ ) of the conflicts.

\section{B. Effect of Arrival Manager on System Performance}

In this section, results are presented that quantify the effect of the Arrival Manager on the overall performance of the integrated system. The performance is presented in terms of both the resolution success rate as well as the efficiency of the solution. Resolution success rate is expressed as the observed (CTAS flight track data) rate of successful resolution of distance-based conflicts. Efficiency is expressed by the number of merging-arrival pairs that violate the one-minute time-in-trail spacing constraint as well as by the magnitude of those violations.

In order to compare how the Arrival Manager would affect the overall system performance, $1 \mathrm{X}$ and $2 \mathrm{X}$ test cases were conducted with the Arrival Manager disabled. These simulation runs emulated the separation assurance system without an integrated time-based metering capability. No time-in-trail spacing constraints were imposed, meaning that time-in-trail spacing and sequencing were not done. Therefore, only distance-based conflicts were detected and resolved.

The resolution success rate results are divided into those related to all conflicts and those related to the mergingarrival conflicts only. These results are shown in Figs. 6a and 6b, respectively. Results indicate that in $1 \mathrm{X}$ and $2 \mathrm{X}$ traffic, the conflict resolution success rates for all aircraft are slightly higher with the Arrival Manager enabled. Success rates for the conflicts among only the merging-arrivals are also higher. These results show that imposing time-in-trail spacing did not adversely affect the resolution success rate of the system.
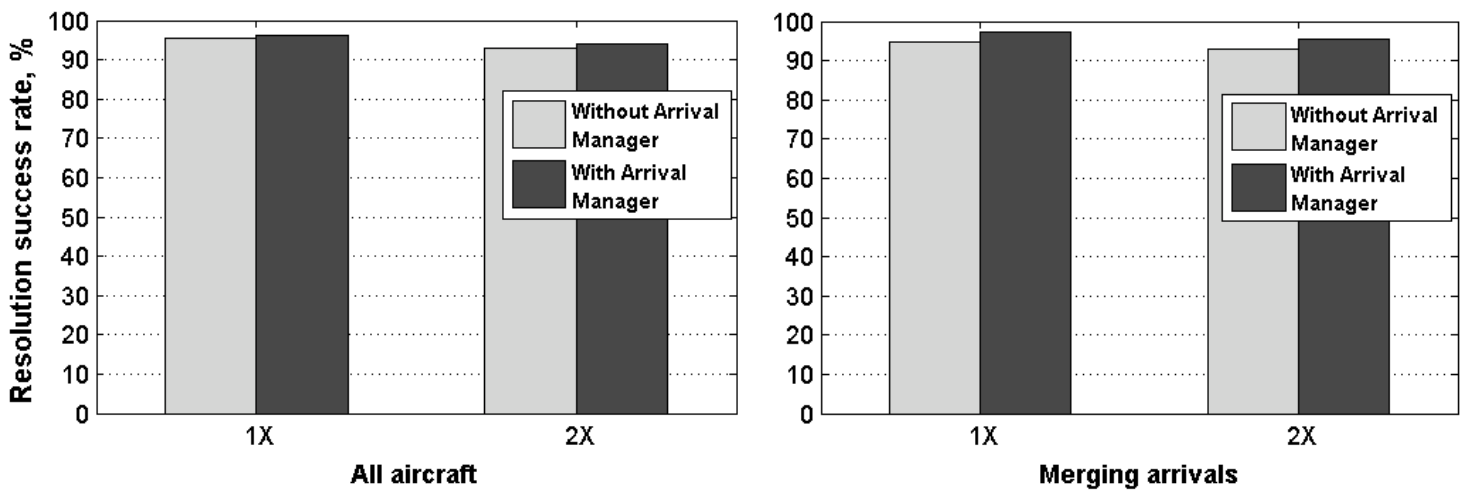

Figure 6. Resolution success rates with and without Arrival Manager for (a) all aircraft and (b) merging arrivals. 
The efficiency aspect of the effect of Arrival Manager is addressed in Figs. 7a and 7b. The histograms show the distribution of the time-in-trail spacing for the 9-hour simulation. For example, the number of time-in-trail spacing constraint violations (e.g. sequencing conflicts) in the (0-14) bin is the percentage of the merging-arrival pairs that are between zero and fourteen seconds apart as they pass through the arrival fix. The bins only go up to 59 seconds because a time-in-trail equal to or above 60 seconds, by definition, is not considered a violation. As seen in both the $1 \mathrm{X}$ and $2 \mathrm{X}$ traffic histograms, both the number and magnitude of the violations are lower with Arrival Manager enabled.
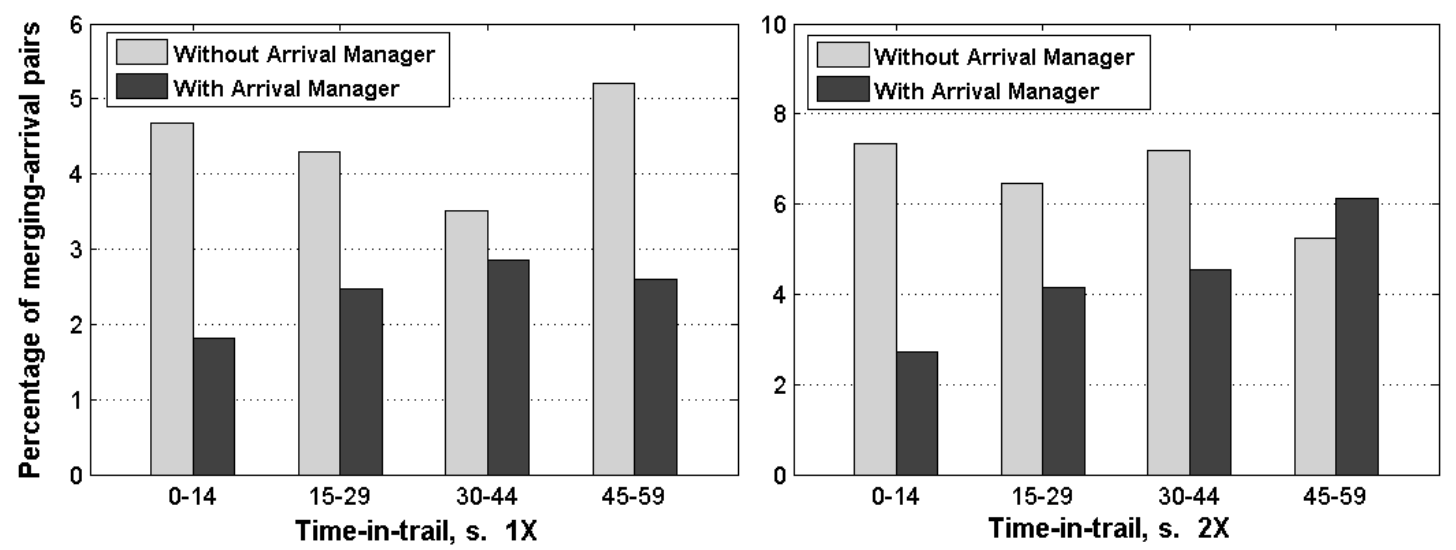

Figure 7. Distribution of time-in-trail spacing violations as a percentage of all in-trail aircraft pairs for the (a) $1 \mathrm{X}$ and (b) $2 \mathrm{X}$ case.

\section{Summary}

The implementation and analysis of the integrated ground-based separation assurance and time-based metering prototype into the Center-TRACON Automation System was presented. The integration of this new capability accommodates constraints in four-dimensions: position (x-y), altitude, and meter-fix crossing time. Experiments were conducted to evaluate the performance of the integrated system and its ability to handle traffic levels of up to twice that of today.

The experimental results were presented in two parts: a) distance-based performance of the integrated system, and b) effect of Arrival Manager on system performance. Results indicate that the integrated system's conflictresolution success rate (distance-based) is over $96 \%$ and $94 \%$ in $1 \mathrm{X}$ and $2 \mathrm{X}$ traffic, respectively. Also in both traffic scenarios, the integrated system was able to identify more than one acceptable resolution for a high percentage (over 90\%) of the conflicts. When comparing the system with and without Arrival Manager, results indicate that at $1 \mathrm{X}$ and $2 \mathrm{X}$ traffic, the distance-based conflict resolution success rates are slightly higher with Arrival Manager. By enabling Arrival Manager, both the number of time-in-trail spacing constraint violations and the magnitude of those violations were reduced. This benefit was achieved without adversely affecting the distance-based conflict resolution success rate of the system. The data suggest that the integrated system is relatively insensitive to an increase in traffic of up to twice the current levels. 


\section{Acknowledgments}

The authors would like to express their gratitude to colleagues and management whose efforts have contributed to this research. Sincere thanks to Todd Farley, Heinz Erzberger, Karl Bilimoria, Salman Suharwardy, and David Thipphavong, for fruitful discussions. Technical support provided by Yung-Cheng Chu and Scott Sahlman is much appreciated.

\section{References}

${ }^{1}$ Department of Transportation, Federal Aviation Administration, Air Traffic Control, Order JO 7110.65S, 14 February 2008, http://www.faa.gov/air_traffic/publications/

${ }^{2}$ Erzrberger, H., "Automated Conflict Resolution for Air Traffic Control," Proceedings of the $25^{\text {th }}$ International Congress of the Aeronautical Sciences (ICAS), Germany, 2006.

${ }^{3}$ Erzberger, H., "Separation Assurance in the Future Air Traffic System," Proceedings of the ENRI International Workshop on ATM/CNS (EIWAC), Tokyo, Japan, 2009.

${ }^{4}$ Erzberger, H., Lauderdale, T.A., Chu, Y.C., "Automated Conflict Resolution, Arrival Management and Weather Avoidance for ATM," Proceedings of the $27^{\text {th }}$ International Congress of the Aeronautical Sciences (ICAS), France, 2010.

${ }^{5}$ Farley, T., Kupfer, M., Erzberger, H., "Automated Conflict Resolution: A Simulation Evaluation Under High Demand Including Merging Arrivals," Proceedings of the $7^{\text {th }}$ AIAA Aviation Technology, Integration and Operations (ATIO) Conference, Belfast, Northern Ireland, 2007.

${ }^{6}$ Kupfer, M., Farley, T., Yung-Cheng, C., and Erzberger, H., "Automated Conflict Resolution: A Simulation-Based Sensitivity Study of Airspace and Demand," Proceedings of the $26^{\text {th }}$ International Congress for the Aeronautical Sciences (ICAS), Anchorage, AK, 2008.

${ }^{7}$ Farley, T. Erzberger, H., "Fast-Time Simulation Evaluation of A Conflict Resolution Algorithm Under High Air Traffic Demand," Proceedings of the $7^{\text {th }}$ USA/Europe ATM R\&D Seminar, Barcelona, Spain, 2007.

${ }^{8}$ Erzberger, H., "Transforming the NAS: The Next Generation Air Traffic Control System," NASA/TP-2004-212828, October 2004.

${ }^{9} \mathrm{McNally}, \mathrm{D} .$, and Gong, C., "Concept and Laboratory Analysis of Trajectory-Based Automation for Separation Assurance," Air Traffic Control Quarterly, Vol. 15(1), pp. 35-63, 2007. 